\title{
CAUCHY PROBLEM AND ANALYTIC CONTINUATION FOR SYSTEMS OF FIRST ORDER ELLIPTIC EQUATIONS WITH ANALYTIC COEFFICIENTS
}

\author{
BY \\ CHUNG-LING YU
}

\begin{abstract}
Let $a, b, c, d, f, g$ be analytic functions of two real variables $x, y$ in the $z=x+i y$ plane. Consider the elliptic equation (M) $\partial u / \partial x-\partial v / \partial y=a u+b v+f$, $\partial u / \partial y+\partial v / \partial x=c u+d v+g$. The following areas will be investigated:

(1) the integral respresentations for solutions of (M) to the boundary $\partial G$ of a simply connected domain $G$;

(2) reflection principles for $(M)$ under nonlinear analytic boundary conditions;

(3) the sufficient conditions for the nonexistence and analytic continuation for the solutions of the Cauchy problem for (M).
\end{abstract}

0. Introduction. In this paper we shall study the integral representations for solutions of the elliptic equations of the following type:

$$
\begin{aligned}
& \partial u / \partial x-\partial v / \partial y=a u+b v+f, \\
& \partial u / \partial x+\partial v / \partial y=c u+d v+g,
\end{aligned}
$$

in a closed domain $\bar{G}$ of the $z=x+i y$ plane, where the coefficients are analytic functions of two real variables $x, y$ in a neighborhood of $\bar{G}$. We also study its applications to the Cauchy problem and the reflection principles under nonlinear analytic boundary conditions for solutions of (0.1).

The theory described in the work of Vekua [8] has established the complex integral representation (1.5) for the solution $w(z)=u+i v$ of $(0.1)$ in a simply connected domain $G$. However, his theorem cannot immediately be applied to $G \cup \partial G$, where $\partial G$ is the boundary of $G$. In $Y u$ [9], the above integral representation (1.5) of $w(z)$ has been extended to $G \cup \partial G$ under some restrictive boundary conditions, namely, $w(z)$ has to be assumed Hölder continuous on $\partial G$. In a variety of applications, due to the generality of the boundary conditions, the above-mentioned representation is not general enough to be applied to some wilder classes of solutions. This is also the main difficulty encountered in applying the method of Lewy [5] to study the reflection principles under nonlinear boundary conditions and Cauchy problem for solutions of (0.1).

We shall overcome this difficulty in $\$ 2$ by an elementary method. Our method is actually new and simpler than the one in [9].

Received by the editors November 14, 1972.

AMS (MOS) subject classifications (1970). Primary 35Cl5, 35J67, 35J45, 35J99, 35F10, 30A92, $30 \mathrm{~A} 93$.

Key words and phrases. First order elliptic equations, pseudo-analytic functions, CauchyRiemann equations, Cauchy problem, analytic continuation, Volterra integral equations.

Copyright $\odot$ 1974, American Mathematical Sociery 
The classical reflection principle for the Cauchy-Riemann equations has been generalized by many authors to solutions of various types of elliptic equations with analytic coefficients in the plane under linear or nonlinear analytic boundary conditions (Lewy [5], [6], Garabedian [4]). Yu [9] has obtained reflection principles for solutions of $(0.1)$ under certain linear analytic boundary conditions and the references are also given there. In this paper we shall further investigate the reflection principle for solutions of $(0.1)$ under the nonlinear analytic boundary conditions which are also generalizations of those given by Lewy [5], [6].

By investigating the relations between $\Delta_{2} u+\lambda u=0$ and $\Delta_{2} u=0$ whose solutions share Cauchy data, Lewy [5] gave very interesting sufficient conditions for the analytic continuation and the nonexistence of the solution of the Cauchy problem for the equation $\Delta_{2} u+\lambda u=0$. We shall generalize Lewy's results by studying the relations between two different differential equations of type (0.1) whose solutions share Cauchy data. We are also able to give several sufficient conditions for the nonexistence and the analytic continuation of the solutions of the Cauchy problem. Furthermore, the domain of continuation is explicitly stated.

In $\$ 1$ we give some definitions and a brief summary of Vekua's theory of the representations of the solutions of equation (0.1) in a domain $G$. In $\$ 2$ we extend the integral representation (1.5) to the boundary of the domain on which the solution is given. In $\$ 3$ we establish the reflection principle for solutions of (0.1) under nonlinear analytic boundary conditions. In $\$ 4$ we study the nonexistence and analytic continuation for the solution of the Cauchy problem for the equation (0.1).

1. Notations and Vekua's integral representations. The notations and definitions which are used in this paper can be found in $\mathrm{Yu}$ [9].

By introducing the complex notation

$$
\partial / \partial \bar{z}=\frac{1}{2}(\partial / \partial x+i \partial / \partial y)
$$

we can write $(0.1)$ in the complex form

$$
\partial w / \partial \bar{z}=A w+B \bar{w}+F
$$

where

$$
\begin{aligned}
& W=u+i v, \quad A=\frac{1}{4}(a+d+i c-i b), \\
& B=\frac{1}{4}(a-d+i c+i b), \quad F=\frac{1}{2}(f+i g) .
\end{aligned}
$$

If we continue $a, b, c, d, f, g$ into the complex, we obtain $A, B, F$ as holomorphic functions of the two complex variables $z=x+i y, \zeta=x-i y$.

From now on we will assume $D$ to be a simply connected domain on the $z=x+i y$ plane whose boundary $\partial D$ is supposed to contain a segment $\sigma$, $\sigma=\{x: a<x<b\}$. We assume $\sigma$ contains the origin as an interior point. 
We also assume $A(z, \zeta), B(z, \zeta), F(z, \zeta)$ are holomorphic functions of the variables $z, \zeta \in D \cup \sigma \cup \bar{D}$, where $\bar{D}=\{z \mid \bar{z} \in D\}$. Set

$$
\begin{aligned}
& C(z, \zeta)=B(z, \zeta) \exp \left[\int_{\zeta_{1}}^{z} A^{*}(\zeta, t) d t-\int_{\zeta}^{\zeta} A(z, t) d t\right], \\
& F_{0}(z, \zeta)=F(z, \zeta) \exp \left[-\int_{\zeta}^{\zeta} A(z, t) d t\right] .
\end{aligned}
$$

Recall that $f^{*}\left(\zeta_{1}, \ldots, \zeta_{n}\right)$ denotes the ${ }^{*}$ conjugate function to a holomorphic function $f\left(z_{1}, \ldots, z_{n}\right)$ and is defined according to the formula

$$
f^{*}\left(\zeta_{1}, \ldots, \zeta_{n}\right)=f\left({\overline{\zeta_{1}}, \ldots, \bar{\zeta}_{n}}\right)
$$

(cf. $\mathrm{Yu}[9, \S 2])$.

We now state Vekua's integral representation for solutions of (1.1) in a simply connected domain $G \subset D \cup \sigma \cup \bar{D}$.

Theorem 1.1. Every $C^{\prime}$ solution $w(z)$ of differential equation (1.1) in $G$ has the integral representation

$$
\begin{aligned}
w(z)=\{ & \phi(z)+\int_{z_{0}}^{z} \Gamma_{1}\left(z, \bar{z}, t, \zeta_{0}\right) \phi(t) d t \\
& \left.+\int_{\zeta_{0}}^{z} \Gamma_{2}\left(z, \bar{z}, z_{0}, \tau\right) \phi^{*}(\tau) d \tau+U_{0}(z, \bar{z})\right\} \exp \int_{\zeta_{1}}^{z} A(z, t) d t
\end{aligned}
$$

where $\left(z_{0}, \zeta_{0}\right)$ is a fixed point in $(G, \bar{G}), \bar{G}=\{z \mid \bar{z} \in G\}, \phi(z)$ is a holomorphic function in $G, U_{0}(z, \zeta)$ is a holomorphic function for $z, \zeta \in D \cup \sigma \cup \bar{D}$, and $\Gamma_{1}, \Gamma_{2}$ are two holomorphic functions of the four variables $z, t, \zeta, \tau \in D \cup \sigma \cup \bar{D}$. Furthermore,

$$
\begin{aligned}
U_{0}(z, \zeta)= & \int_{\zeta_{0}}^{\zeta} F_{0}(z, \tau) d \tau \\
& +\int_{\zeta 0}^{\zeta} d \tau \int_{z_{0}}^{z} \Gamma_{1}(z, \zeta, t, \tau) F_{0}(t, \tau) d t \\
& +\int_{\zeta_{0}}^{\zeta} d \tau \int_{z_{0}}^{z} \Gamma_{2}(z, \zeta, t, \tau) F_{0}^{*}(t, \tau) d t
\end{aligned}
$$

and

$$
\begin{aligned}
& \Gamma_{1}(z, \zeta, t, \tau)=\int_{\tau}^{\zeta} \Gamma(z, \zeta, t, \eta) d \eta \\
& \Gamma_{2}(z, \zeta, t, \tau)=C(z, \tau)+\int_{t}^{z} C(\xi, \tau) \Gamma_{1}(z, \zeta, \xi, \tau) d \xi,
\end{aligned}
$$

where $\Gamma$ is the unique holomorphic solution of the Volterra integral equation 


$$
\begin{aligned}
\Gamma(z, \zeta, t, \tau)= & C(z, \tau) C^{*}(\tau, t) \\
& +\int_{\tau}^{\zeta} d \eta \int_{t}^{z} C(\xi, \tau) C^{*}(\tau, t) \Gamma(z, \tau, \xi, \eta) d \xi,
\end{aligned}
$$

for $z, \zeta, t, \tau \in D \cup \sigma \cup \bar{D}$.

Conversely, the function $w(z)$ which is given by the formula (1.5) is a solution of differential equation (1.1) in $G$.

\section{Integral representations on $G \cup \partial G$.}

Lemma 2.1. Let $G$ be a simply connected domain in the complex plane. If $w(z)$ is uniform continuous on $G$, then there exists a unique continuous function $g(z)$ on $G \cup \partial G$ such that $g(z)=w(z)$ on $G$, where $\partial G$ denotes the boundary of $G$.

Proof. See Graves [2, p. 117].

Theorem 2.1. Every $C^{\prime}$ solution $w(z)$ of differential equation $(1.1)$ in $D$, continuous in $D \cup \sigma$, has the integral representation (1.5) where the function $\phi(z)$ is holomorphic in $D$, and can be defined as a continuous function on $D \cup \sigma$.

Proof. Let $D_{0}$ be an arbitrary open half disc in $D$, whose boundary $\partial D_{0}$ contains a segment $\sigma_{0}, \sigma_{0} \subset \sigma$, and $\partial D_{0} \cup D_{0} \subset D \cup \sigma$. It suffices to prove that $\phi(z)$ is continuous in $D_{0} \cup \partial D_{0}$.

According to (1.5), $w(z)$ has representation

$$
\begin{aligned}
w(z)=\{ & \phi(z)+\int_{z_{0}}^{z} \Gamma_{1}\left(z, \bar{z}, t, \bar{z}_{0}\right) \phi(t) d t \\
& \left.+\int_{\bar{z}_{0}}^{z} \Gamma_{2}\left(z, \bar{z}, z_{0}, \tau\right) \phi^{*}(\tau) d \tau+U_{0}(z, \bar{z})\right\} \exp \left[\int_{\zeta_{1}}^{z} A(z, t) d t\right] .
\end{aligned}
$$

Let $G$ be a simply connected compact subset of $D \cup \sigma$ which contains $D_{0}$ and $z_{0}$. Then $\left|\Gamma_{1}(z, \zeta, t, \tau)\right|$ and $\left|\Gamma_{2}(z, \zeta, t, \tau)\right|$ are bounded by a constant $M$ for $z, t \in G$, $\zeta, \tau \in \bar{G}$.

Let $z_{1}, z_{2}$ be any two points in $D_{0}$. Also let $\overline{z_{0} z_{1}}, \overline{z_{1} z_{2}}$ denote segments from $z_{0}$ to $z_{1}$ and $z_{1}$ to $z_{2}$ respectively. Let

$$
k(z)=w(z) \exp \left[-\int_{\xi_{1}}^{\bar{z}} A(z, t) d t\right]-U_{0}(z, \bar{z}) ;
$$

therefore, (2.1) becomes

$$
\begin{aligned}
k(z)= & \phi(z)+\int_{z_{0}}^{z} \Gamma_{1}\left(z, \bar{z}, t, \bar{z}_{0}\right) \phi(t) d t \\
& +\int_{\bar{z}_{0}}^{\bar{z}} \Gamma_{2}\left(z, \bar{z}, z_{0}, \tau\right) \phi^{*}(\tau) d \tau
\end{aligned}
$$

for $z \in \overline{z_{1} z_{2}} \cup \overline{z_{0} z_{1}}$.

In view of the method of successive approximation, we have 


$$
\phi(z)=k(z)+\int_{z_{0}}^{z} H_{1}(z, t) k(t) d t+\int_{z_{0}}^{z} H_{2}(z, t) k^{*}(t) d t
$$

for $z \in \overline{z_{1} z_{2}} \cup \overline{z_{0} z_{1}}$. Furthermore,

$$
\begin{array}{ll}
\left|H_{1}(z, t)\right| \leq 4 M \exp (4 M|z-t|)<M_{1}, & z, t \in \overline{z_{1} z_{2}}, \\
\left|H_{2}(z, t)\right| \leq 4 M \exp (4 M|z-t|)<M_{1}, & z, t \in \overline{z_{1} z_{2}}
\end{array}
$$

where $M_{1}$ is a constant independent of $k, \phi, z_{1}, z_{2}$. Since

$$
\begin{aligned}
\phi\left(z_{2}\right)-\phi\left(z_{1}\right)= & \left(z_{2}\right)-k\left(z_{1}\right)+\int_{z_{1}}^{z_{2}} H_{1}(z, t) k(t) d t \\
& +\int_{\bar{z}_{1}}^{\bar{z}_{2}} H_{2}(z, t) k^{*}(t) d t,
\end{aligned}
$$

we have

$$
\left|\phi\left(z_{2}\right)-\phi\left(z_{1}\right)\right| \leq\left|k\left(z_{2}\right)-k\left(z_{1}\right)\right|+2 M_{1} p\left|z_{2}-z_{1}\right|
$$

where $p=\max _{z \in G}|k(z)|$.

Since $k(z)$ is uniform continuous in $D_{0}, p$ and $M_{1}$ are independent of $z_{1}$ and $z_{2}$, by (2.4), $\phi(z)$ is uniform continuous on $D_{0}$; therefore, $\phi(z)$ has continuous extension to $D_{0} \cup \partial D_{0}$ (Lemma 2.1). This completes the proof.

Lemma 2.2. If $K(z, t), f(t)$ are holomorphic for $z, t \in D \cup \sigma \cup \bar{D}$, then

$$
\int_{0}^{2} K(2, t) f(t) d t=f(0) \int_{0}^{2} K(z, t) d t+\int_{0}^{2} K_{1}(2, t) f^{\prime}(t) d t
$$

where $K_{1}(z, t)=\int_{t}^{z} K\left(z, t^{\prime}\right) d t^{\prime}$.

Theorem 2.2. Every solution $w(z)$ of differential equation (1.1) in $D, C^{\prime}$ in $D \cup \sigma$, has the integral representation (1.5), where the function $\phi(z)$ is holomorphic in $D$ and can be defined as $a C^{\prime}$ function on $D \cup \sigma$.

Proof. It is clear that $w(z)$ is $C^{\prime}$ in $D \cup \sigma$ if and only if $w_{0}(z)$ is $C^{\prime}$ in $D \cup \sigma$, where

$$
w(z)=w_{0}(z) \exp \left[\int_{\zeta_{1}}^{\bar{z}} A(z, t) d t\right] .
$$

By (1.5), $w_{0}(z)$ has representation

$$
\begin{aligned}
w_{0}(z)= & \phi(z)+\int_{z_{0}}^{z} \Gamma_{1}\left(z, \bar{z}, t, \bar{z}_{0}\right) \phi(t) d t \\
& +\int_{\bar{z}_{0}}^{\bar{z}} \Gamma_{2}\left(z, \bar{z}, z_{0}, \tau\right) \phi^{*}(\tau) d \tau+U_{0}(z, \bar{z}) .
\end{aligned}
$$


Differentiating (2.5), we have

$$
\begin{aligned}
\partial w_{0} / \partial z= & \phi^{\prime}(z)+\Gamma_{1}\left(z, \bar{z}, z, \bar{z}_{0}\right) \phi(z) \\
& +\int_{z_{0}}^{z} \Gamma_{1, z}\left(z, \bar{z}, t, \bar{z}_{0}\right) \phi(t) d t \\
& +\int_{\bar{z}_{0}}^{\bar{z}} \Gamma_{2, z}\left(z, \bar{z}, z_{0}, \tau\right) \phi^{*}(\tau) d \tau+U_{0, z}(z, \bar{z})
\end{aligned}
$$

where $\Gamma_{1, z}\left(z, \bar{z}, t, z_{0}\right)=(\partial / \partial z) \Gamma_{1}\left(z, \bar{z}, t, z_{0}\right), \ldots$, etc.

By Lemma 2.2, (2.6) can be rewritten as the following:

$$
\begin{aligned}
\frac{\partial w_{0}}{\partial z}= & \phi^{\prime}(z)+\int_{z_{0}}^{z} H_{1}(z, t) \phi^{\prime}(t) d t \\
& +\int_{\bar{z}_{0}}^{\bar{z}} H_{2}(z, t) \phi^{*^{\prime}}(t) d t+U(z)
\end{aligned}
$$

where $H_{1}(z, t), H_{2}(z, t), U(z)$ are certain continuous functions for $z, t \in D \cup \sigma$ $\cup \bar{D}$.

Since $\partial w_{0} / \partial z$ is continuous in $D \cup \sigma$, by the same argument as Theorem 2.1, $\phi^{\prime}(z)$ has continuous extension in $D \cup \sigma$. This completes the proof.

By the similar argument we have the following generalization.

Theorem 2.3. Let $G$ be a simply connected domain whose boundary $\partial G$ is a piecewise smooth, simple, closed curve and $G \cup \partial G \subset D \cup \sigma \cup \bar{D}$; then every solution $w(z)$ of differential equation (1.1) in $G$, continuous (or $C^{\prime}$ ) in $G \cup \partial G$, has the integral representation (1.5), where the function $\phi(z)$ is holomorphic in $G$, and can be defined as a continuous (or $C^{\prime}$ ) function on $G \cup \partial G$.

3. Reflection principles. The approach to be used in this section is patterned after the ideas of Lewy [5].

Theorem 3.1. Let $w(x, y)=u(x, y)+i v(x, y)$ be a solution of $(1.1)$ in the domain $D$, continuous with its first derivatives in $D \cup \sigma$. Assume that $u(x, y)$ satisfies on $y=0$ a real relation

$$
u_{y}=h\left(x, u, v, u_{x}\right)
$$

where $h$ is an analytic function of its arguments near $x=0, u=u(0,0), v$ $=v(0,0), u_{x}=u_{x}(0,0)$. Then $w(x, y)$ can be continued analytically across $y=0$ near the origin.

Proof. Introducing the differential operators

$$
\frac{\partial}{\partial z}=\frac{1}{2}\left(\frac{\partial}{\partial x}-i \frac{\partial}{\partial y}\right), \quad \frac{\partial}{\partial \bar{z}}=\frac{1}{2}\left(\frac{\partial}{\partial x}+i \frac{\partial}{\partial y}\right),
$$


(3.1) becomes $u_{z}=u_{z}+i h\left(x, u, v, u_{z}+u_{z}\right)$ which can be solved algebraically with respect to $u_{z}$ since $1-i \partial h / \partial u_{x} \neq 0$. Therefore, we can assume $u_{z}$ in terms of $u_{z}$, $u, v$ the following form

$$
u_{\bar{z}}=H_{1}\left(x, u, v, u_{z}\right) \text {. }
$$

Similarly we can assume $u_{z}$ the following form

$$
u_{z}=H_{2}\left(x, u, v, u_{\mathbf{z}}\right) \text {. }
$$

Note that $H_{1}$ and $H_{2}$ are holomorphic functions in the neighborhoods of $\left(0, u(0,0), v(0,0), u_{z}(0,0)\right)$ and $\left(0, u(0,0), v(0,0), u_{\bar{z}}(0,0)\right)$ respectively.

Set

$$
\begin{aligned}
U(z, \bar{z}) & =u(z)=\frac{1}{2}(w+\bar{w}), \\
V(z, \bar{z}) & =v(z)=(1 / 2 i)(w-\bar{w}), \\
F(z, \zeta) & =\exp \int_{\zeta_{1}}^{\zeta} A(z, t) d t,
\end{aligned}
$$

so that, by Theorem 2.2 , we have

$$
\begin{aligned}
& 2 U(z, \zeta)=\{F(z, \zeta) \phi(z) \\
&+F(z, \zeta) \int_{z_{0}}^{z} \Gamma_{1}\left(z, \zeta, t, \bar{z}_{0}\right) \phi(t) d t \\
&\left.+F(z, \zeta) \int_{\bar{z}_{0}}^{\zeta} \Gamma_{2}\left(z, \zeta, z_{0}, \tau\right) \phi^{*}(\tau) d \tau+U_{0}(z, \zeta) F(z, \zeta)\right\} \\
&+\left\{F^{*}(\zeta, z) \phi^{*}(\zeta)+F^{*}(\zeta, z) \int_{\bar{z}_{0}}^{\zeta} \Gamma_{1}^{*}\left(\zeta, z, t, z_{0}\right) \phi^{*}(t) d t\right. \\
&\left.+F^{*}(\zeta, z) \int_{z_{0}}^{z} \Gamma_{2}^{*}\left(\zeta, z, \bar{z}_{0}, \tau\right) \phi(\tau) d \tau+U_{0}^{*}(\zeta, z) F^{*}(\zeta, z)\right\} .
\end{aligned}
$$

Recall that $\Gamma_{1}\left(z, \zeta, t, \bar{z}_{0}\right), \Gamma_{2}\left(z, \zeta, z_{0}, \tau\right), \Gamma_{1}^{*}\left(z, \zeta, t, z_{0}\right), \Gamma_{2}^{*}\left(\zeta, z, \bar{z}_{0}, t\right), F(z, \zeta)$. $U_{0}(z, \zeta), F^{*}(\zeta, z), U_{0}^{*}(\zeta, z)$ are holomorphic for $z, \zeta \in D \cup \sigma \cup \bar{D}, \phi(z)$ is a function holomorphic in $D$ and can be defined as a continuous differentiable function in $D \cup \sigma$.

Differentiating (3.7), we have

$$
\begin{aligned}
U_{z}(z, \zeta)= & F(z, \zeta) \phi^{\prime}(z)+\int_{0}^{z} U_{1}(z, \zeta, t) \phi^{\prime}(t) d t \\
& +\int_{\bar{z}_{0}}^{\zeta} U_{2}(z, \zeta, t) \phi^{*}(t) d t+\alpha_{1}(z, \zeta)
\end{aligned}
$$

where $U_{1}(z, \zeta, t), U_{2}(z, \zeta, t), \alpha_{1}(z, \zeta)$ are certain analytic functions of $z, \zeta, t$ $\in D \cup \sigma \cup \bar{D}$. 
Similarly, we have

$$
\begin{aligned}
U_{\zeta}(z, \zeta)= & F^{*}(\zeta, z) \phi^{* \prime}(\zeta)+\int_{0}^{\zeta} U_{3}(z, \zeta, t) \phi^{* \prime}(t) d t \\
& +\int_{z_{0}}^{z} U_{4}(z, \zeta, t) \phi(t) d t+\alpha_{2}(z, \zeta)
\end{aligned}
$$

where $U_{3}(z, \zeta, t), U_{4}(z, \zeta, t), \alpha_{2}(z, \zeta)$ are certain analytic functions of $z, \zeta, t$ $\in D \cup \sigma \cup \bar{D}$.

By Lemma 2.2, we can rewrite (3.7) as

$$
U(z, \zeta)=\int_{0}^{z} U_{5}(z, \zeta, t) \phi^{\prime}(t) d t+\alpha_{3}(z, \zeta)
$$

where $U_{5}(z, \zeta, t)$ is holomorphic in $D \cup \sigma \cup \bar{D}, \alpha_{3}(z, z)$ is holomorphic in $\bar{D}$ and continuous in $\bar{D} \cup \sigma$.

Similarly

$$
V(z, \zeta)=\int_{0}^{z} U_{6}(z, \zeta, t) \phi^{\prime}(t) d t+\alpha_{4}(z, \zeta)
$$

and

$$
U_{\zeta}(z, \zeta)=\int_{0}^{z} U_{7}(z, \zeta, t) \phi^{\prime}(t) d t+\alpha_{5}(z, \zeta)
$$

where $U_{6}(z, \zeta, t), U_{7}(z, \zeta, t)$ are holomorphic in $D \cup \sigma \cup \bar{D}, \alpha_{4}(z, z)$ and $\alpha_{5}(z, z)$ are holomorphic in $\bar{D}$ and continuous in $\bar{D} \cup \boldsymbol{\sigma}$.

Define the operators $A_{i}, i=1,2,3$, by

$$
\begin{aligned}
& A_{1}(f)(z)=\int_{0}^{z} U_{5}(z, z, t) f(t) d t+\alpha_{3}(z, z), \\
& A_{2}(f)(z)=\int_{0}^{z} U_{6}(z, z, t) f(t) d t+\alpha_{4}(z, z), \\
& A_{3}(f)(z)=\int_{0}^{z} U_{7}(z, z, t) f(t) d t+\alpha_{5}(z, z) ;
\end{aligned}
$$

then we see $A_{1}\left(\phi^{\prime}\right)(z)=U(z, z), A_{2}\left(\phi^{\prime}\right)=V(z, z), A_{3}\left(\phi^{\prime}\right)=U_{z}(z, z)$.

Substituting the above formulas into (3.3), we have for $z \in \sigma$,

$$
\begin{aligned}
& \phi^{\prime}(z)=k(z)+\left\{H_{2}\left(z, A_{1} \phi^{\prime}, A_{2} \phi^{\prime}, A_{3} \phi^{\prime}\right)\right. \\
&\left.-\int_{0}^{z} U_{1}(z, z, t) \phi^{\prime}(t) d t-U_{z}(0,0)\right\} / F(z, z) \\
&=k(z)+T \phi^{\prime}
\end{aligned}
$$

where

$$
k(z)=\left\{-\int_{z_{0}}^{z} U_{2}(z, z, t) \phi^{*}(t) d t-\alpha_{1}(z, z)+U_{z}(0,0)\right\} / F(z, z) .
$$


We now proceed to use the contraction mapping principle to find a solution of (3.16) in $(|z|<\rho) \cap(\bar{D} \cup \sigma)$ where $\rho$ is a sufficiently small constant.

The operator $T$ is well defined on $\bar{D} \cup \sigma$, since $T$ depends on $\phi^{*}(t)$ and we know that $\phi^{*}(t)$ is holomorphic in $\bar{D}$ and continuous in $\bar{D} \cup \sigma$. Moreover, $\left(T \phi^{\prime}\right)(0)=0$.

In view of the analyticity of $\mathrm{H}_{2}$, there is a small circle $\gamma$ with center 0 and a constant $M_{1}$ such that for $\left|\phi^{\prime}(z)\right| \leq M_{1}, z \in \gamma \cap(\bar{D} \cup \sigma), H_{2}$ is holomorphic for its arguments.

If the circle $\gamma$ has sufficiently small radius, from (3.8) we see $|k(z)|<M_{1}$ for $z \in \gamma \cap(\bar{D} \cup \sigma)$.

The function $\mathrm{H}_{2}$ satisfies a Lipschitz condition with respect to the last 3 arguments. Hence, for $z \in \gamma \cap(\bar{D} \cup \sigma)$,

$$
\begin{aligned}
|1 / F(z, z)| \mid & H_{2}\left(z, A_{1} \phi_{1}, A_{2} \phi_{1}, A_{3} \phi_{1}\right)-H_{2}\left(z, A_{1} \phi_{2}, A_{2} \phi_{2}, A_{3} \phi_{2}\right) \mid \\
\leq & C_{0}\left\{\left|A_{1} \phi_{1}-A_{1} \phi_{2}\right|+\left|A_{2} \phi_{1}-A_{2} \phi_{2}\right|+\left|A_{3} \phi_{1}-A_{3} \phi_{2}\right|\right\} .
\end{aligned}
$$

For a given $a, 0 \leq a<1$, and $\rho$ sufficiently small, it is easily seen that

$$
\begin{gathered}
\sup _{z \in G}\left|A_{i} \phi_{1}-A_{i} \phi_{2}\right| \leq \frac{a}{4 C_{0}} \sup _{z \in G}\left|\phi_{1}-\phi_{2}\right|, \quad i=1,2,3, \\
\sup _{z \in G}\left|\frac{1}{F} \int_{0}^{z} U_{1}(z, z, t) \phi_{1}(t) d t-\frac{1}{F} \int_{0}^{z} U_{1}(z, z, t) \phi_{2}(t) d t\right| \\
\leq \frac{a}{4 C_{0}} \sup _{z \in G}\left|\phi_{1}-\phi_{2}\right|
\end{gathered}
$$

where $G=\{|z|<\rho\} \cap\{\bar{D} \cup \sigma\}$. Hence

$$
\sup _{z \in G}\left|T \phi_{1}-T \phi_{2}\right| \leq a \sup _{z \in G}\left|\phi_{1}-\phi_{2}\right|
$$

Therefore by method of successive approximation, there exists a unique solution $\phi^{\prime}(z)$ for (3.16) in $\{|z|<\rho\} \cap(\bar{D} \cup \sigma)$. This solution is unique and continuous on $\{|z|<\rho\} \cap(\bar{D} \cup \sigma)$, and is regular in $(|z|<\rho) \cap \bar{D}$. But on $\sigma$, (3.16) expresses the boundary condition which the given $u(x, y)$ satisfies by hypothesis. Hence on $\sigma,(3.16)$ holds for $\phi^{\prime}(z)$ as just constructed as for $\phi^{\prime}(z)$ as given by (1.5). The two determinations are equal on account of uniqueness. Therefore, we have obtained an analytic continuation $\phi^{\prime}(z)$ from $D$ into $(|z|<\rho)$ $\cap(\bar{D} \cup \boldsymbol{\sigma})$.

Now we know that $\phi(z)$ of (1.5) is analytic in a neighborhood of the origin. This completes the proof.

4. Nonexistence and analytic continuation for the solutions of the Cauchy problem. Let us consider the following two different equations of type (1.1):

$$
\partial w / \partial \bar{z}=A_{1}(z, \bar{z}) w+B_{1}(z, \bar{z}) \bar{w}+F_{1}(z, \bar{z})
$$


and

$$
\partial w / \partial \bar{z}=A_{2}(z, \bar{z}) w+B_{2}(z, \bar{z}) \bar{w}+F_{2}(z, \bar{z}),
$$

where $A_{i}(z, \zeta), B_{i}(z, \zeta), F_{i}(z, \zeta)$ are holomorphic for $z, \zeta \in D \cup \sigma \cup \bar{D}, i=1$, 2.

The following are two theorems on Cauchy data admitting a solution for (4.1) and for (4.2), which yield sufficient conditions for analytic continuation and the nonexistence of the solutions of (4.1) and (4.2).

Theorem 4.1. Let $w_{1}(x, y)$ be a solution of $(4.1)$ in $D, C^{\prime}$ in $D \cup \sigma, w_{2}(x, y)$ be a solution of (4.2) in $D, C^{\prime}$ in $D \cup \sigma$, such that $w_{1}(x, 0)=w_{2}(x, 0)=\rho(x)$ on $\sigma$. If $B_{1}(x, x) \not B_{2}(x, x), x \in \sigma$, then $\rho(x)$ is analytic on $\sigma$, and its analytic continuation $\rho(z)$ is holomorphic in $D \cup \sigma \cup \bar{D}$, except for the zeros of $B_{1}^{*}(z, z)-B_{2}^{*}(z, z)$ in $\bar{D}$. Furthermore, $w_{1}(x, y)$ and $w_{2}(x, y)$ can be continued analytically into whole $D \cup \sigma$ $\cup \bar{D}$, except for the zeros of $B_{1}^{*}(z, z)-B_{2}^{*}(z, z)$ in $\bar{D}$, as solutions of (4.1) and (4.2) respectively.

Theorem 4.2. If $w_{1}(x, y)$ is a solution of $(4.1)$ in $D$, continuous in $D \cup \sigma, w_{2}(x, y)$ is a solution of (4.2) in $\bar{D}$, continuous in $\bar{D} \cup \sigma$, such that $w_{1}(x, 0)=w_{2}(x, 0)$ $=\rho(x)$ on $\sigma$, then $\rho(x)$ is analytic on $\sigma$, and its analytic continuation $\rho(z)$ is holomorphic in $D \cup \sigma \cup \bar{D}$. Furthermore, $w_{1}(x, y)$ and $w_{2}(x, y)$ can be continued analytically into whole $D \cup \sigma \cup \bar{D}$ as solutions of (4.1) and (4.2) respectively.

The assumption $B_{1}(x, x) \not \equiv B_{2}(x, x), x \in \sigma$, in Theorem 4.1 is indispensable; we shall give an example (Example 4.1 below) to show the analog of Theorem 4.1 for $B_{1}(x, x) \equiv B_{2}(x, x)$ is incorrect.

Proof of Theorem 4.1. According to Theorem 2.2, $w_{i}(z)$ has the representation

$$
\begin{aligned}
w_{i}(z)=\{ & \phi_{i}(z)+\int_{z_{i}}^{z} \Gamma_{1, i}\left(z, \bar{z}, t, \bar{z}_{i}\right) \phi_{i}(t) d t \\
& \left.+\int_{z_{i}}^{z} \Gamma_{2, i}\left(z, \bar{z}, z_{i}, \tau\right) \phi_{i}^{*}(\tau) d \tau+U_{0, i}(z, \bar{z})\right\} \exp \int_{\xi}^{z} A_{i}(z, t) d t,
\end{aligned}
$$

$$
i=1,2 \text {, }
$$

where $\phi_{i}(z)$ is a function holomorphic in $D$ and can be defined as a continuous differentiable function in $D \cup \sigma$. Therefore, for $x \in \sigma$, (4.3) becomes

$$
\begin{aligned}
\rho(x)=\{ & \phi_{i}(x)+\int_{0}^{x} \Gamma_{1, i}\left(x, x, t, \bar{z}_{i}\right) \phi_{i}(t) d t \\
& +\int_{0}^{x} \Gamma_{2, i}\left(x, x, z_{i}, t\right) \phi_{i}^{*}(t) d t \\
& +\int_{z_{i}}^{0} \Gamma_{1, i}\left(x, x, t, \bar{z}_{i}\right) \phi_{i}(t) d t \\
& \left.+\int_{\bar{z}_{i}}^{0} \Gamma_{2, i}\left(x, x, z_{i}, t\right) \phi_{i}^{*}(t) d t+U_{0, i}(x, x)\right\} \exp \int_{\xi}^{x} A_{i}(x, t) d t,
\end{aligned}
$$




$$
\begin{aligned}
\rho^{*}(x)=\{ & \phi_{i}^{*}(x)+\int_{0}^{x} \Gamma_{i, i}^{*}\left(x, x, t, z_{i}\right) \phi_{i}^{*}(t) d t \\
& +\int_{0}^{x} \Gamma_{z_{i}}^{*}\left(x, x, \bar{z}_{i}, t\right) \phi_{i}(t) d t \\
& +\int_{\bar{z}_{i}}^{0} \Gamma_{i, i}^{*}\left(x, x, t, z_{i}\right) \phi_{i}^{*}(t) d t \\
& \left.+\int_{z_{i}}^{0} \Gamma_{z_{i}}^{*}\left(x, x, \bar{z}_{i}, t\right) \phi_{i}(t) d t+U_{0, i}^{*}(x, x)\right\} \exp \int_{\bar{z}}^{x} A_{i}^{*}(x, t) d t,
\end{aligned}
$$

where $\Gamma_{1, i}\left(z, z, t, \bar{z}_{i}\right), \Gamma_{2, i}\left(z, z, z_{i}, t\right), \Gamma_{1, i}^{*}\left(z, z, t, z_{i}\right), \Gamma_{2, i}^{*}\left(z, z, \bar{z}_{i}, t\right), U_{0, i}(z, z), U_{0, i}^{*}(z, z)$, $A_{i}(z, t), A_{i}^{*}(z, t)$ are holomorphic for $z, t \in D \cup \sigma \cup \bar{D}$.

Define $G_{i}(z)=\int_{\xi}^{z} A_{i}(z, t) d t$; thus (4.4) and (4.5) suggest the following integral equations:

$$
\begin{gathered}
\phi_{1}(z) G_{1}(z)-\phi_{2}(z) G_{2}(z)+\int_{0}^{z} \Gamma_{1,1}\left(z, z, t, \bar{z}_{1}\right) G_{1}(z) \phi_{1}(t) d t \\
\quad-\int_{0}^{z} \Gamma_{1,2}\left(z, z, t, \bar{z}_{2}\right) G_{2}(z) \phi_{2}(t) d t=a_{1}(z), \\
\int_{0}^{z} G_{1}^{*}(z) \Gamma_{2,1}^{*}\left(z, z, \bar{z}_{1}, t\right) \phi_{1}(t) d t \\
\quad-\int_{0}^{z} G_{2}^{*}(z) \Gamma_{2,2}^{*}\left(z, z, \bar{z}_{2}, t\right) \phi_{2}(t) d t=a_{2}(z)
\end{gathered}
$$

for the unknown functions $\phi_{1}(z)$ and $\phi_{2}(z)$ in $\bar{D} \cup \sigma$, where

$$
\begin{aligned}
a_{1}(z)= & \int_{\bar{z}_{2}}^{z} \Gamma_{2,2}\left(z, z, z_{2}, t\right) G_{2}(z) \phi_{2}^{*}(t) d t \\
& -\int_{\bar{z}_{1}}^{z} \Gamma_{2,1}\left(z, z, z_{1}, t\right) G_{1}(z) \phi_{1}^{*}(t) d t \\
& +\int_{z_{2}}^{0} \Gamma_{1,2}\left(z, z, t, \bar{z}_{2}\right) G_{2}(z) \phi_{2}(t) d t \\
& -\int_{z_{1}}^{0} \Gamma_{1,1}\left(z, z, t, \bar{z}_{1}\right) G_{1}(z) \phi_{1}(t) d t \\
& +G_{2}(z) U_{0,2}(z, z)-G_{1}(z) U_{0,1}(z, z), \\
a_{2}(z)= & G_{2}^{*}(z) \phi_{2}^{*}(z)-G_{1}^{*}(z) \phi_{1}^{*}(z) \\
& +\int_{\bar{z}_{2}}^{z} \Gamma_{1,2}^{*}\left(z, z, t, z_{2}\right) G_{2}^{*}(z) \phi_{2}^{*}(t) d t \\
& -\int_{\bar{z}_{1}}^{z} \Gamma_{1,1}^{*}\left(z, z, t, z_{1}\right) G_{1}^{*}(z) \phi_{1}^{*}(t) d t \\
& +\int_{z_{2}}^{0} \Gamma_{z_{2}, 2}^{*}\left(z, z, \bar{z}_{2}, t\right) G_{2}^{*}(z) \phi_{2}(t) d t \\
& -\int_{z_{1}}^{0} \Gamma_{z_{1}, 1}^{*}\left(z, z, \bar{z}_{1}, t\right) G_{1}^{*}(z) \phi_{1}(t) d t \\
& +G_{2}^{*}(z) U_{0,2}^{*}(z, z)-G_{1}^{*}(z) U_{0,1}^{*}(z, z) .
\end{aligned}
$$


Recall that

$$
\begin{aligned}
& C_{1}(z, \zeta)=B_{1}(z, \zeta) \exp \left[\int_{\bar{\zeta}_{1}}^{z} A_{1}^{*}(\zeta, t) d t-\int_{\zeta_{1}}^{\zeta} A_{1}(z, t) d t\right] \\
& C_{2}(z, \zeta)=B_{2}(z, \zeta) \exp \left[\int_{\bar{\zeta}_{2}}^{z} A_{2}^{*}(\zeta, t) d t-\int_{\zeta_{2}}^{\zeta} A_{2}(z, t) d t\right] .
\end{aligned}
$$

By (1.7) and (1.8), we see

$$
\begin{aligned}
& \left.\Gamma_{2, i}\right|_{\zeta=\tau}=\Gamma_{2, i}(z, \tau, t, \tau)=C_{i}(z, \tau), \\
& \left.\Gamma_{2, i}\right|_{z=t}=\Gamma_{2, i}(t, \zeta, t, \tau)=C_{i}(t, \tau) .
\end{aligned}
$$

By $B_{1}(x, x)=B_{2}(x, x)$, we have

$$
\begin{aligned}
\Delta(x) & \neq\left|\begin{array}{cc}
G_{1}(x) & G_{2}(x) \\
G_{1}^{*}(x) \Gamma_{2,1}^{*}\left(x, x, \bar{z}_{1}, x\right) & G_{2}^{*}(x) \Gamma_{2,2}^{*}\left(x, x, \bar{z}_{2}, x\right)
\end{array}\right| \\
& =\left\{B_{2}^{*}(x, x)-B_{1}^{*}(x, x)\right\} \exp \left[\int_{\zeta_{2}}^{x} A_{2}(x, t) d t+\int_{\xi_{1}}^{x} A_{1}(x, t) d t\right] \not \equiv 0,
\end{aligned}
$$

for $x \in \sigma$. In particular, if $B_{1}^{*}(z, z)-B_{2}^{*}(z, z) \neq 0$ for $z \in \bar{D}$, then $\Delta(z) \neq 0$ for $z \in \bar{D}$.

Therefore, the integral equations (4.6) and (4.7) have a unique solution $\left(\phi_{1}(z), \phi_{2}(z)\right)$ which is meromorphic in $\bar{D}$, continuous in $\bar{D} \cup \sigma$ (except the zeros of $B_{1}^{*}(z, z)-B_{2}^{*}(z, z)$ in $\left.\bar{D}\right)$, and agree with the given $\left(\phi_{1}(z), \phi_{2}(z)\right)$ on $\sigma$.

Consequently, the holomorphic functions $\phi_{1}(z), \phi_{2}(z)$ in $D$ can be defined as meromorphic functions in $D \cup \sigma \cup \bar{D}$. In the case that $\Delta(z) \neq 0$ for $z \in \bar{D}$, $\phi_{1}(z)$ and $\phi_{2}(z)$ are holomorphic throughout $D \cup \sigma \cup \bar{D}$.

Finally, we return to Theorem $1.1 ; w_{1}(z)$ and $w_{2}(z)$ can be defined as solutions of (4.1) and (4.2) in $D \cup \sigma \cup \bar{D}$ respectively, except the zeros of $B_{1}^{*}(z, z)$ - $B_{2}^{*}(z, z)$ in $\bar{D}$. This completes the proof.

The following example shows that the analog of Theorem 4.1 for $B_{1}(x, x)$ $\equiv B_{2}(x, x)$ is incorrect.

Example 4.1. Let $f(z)$ be holomorphic in $D$, continuous in $D \cup \sigma$, and nonanalytic on $\sigma$. Also let

$$
W(z, \zeta)=f(z) \exp \left[\int_{0}^{\zeta} A(z, t) d t-\int_{0}^{z} A(z, t) d t\right] .
$$

It is clear that $w(x, y)=W(z, \bar{z})$ is a solution of $\partial w / \partial \bar{z}=A(z, \bar{z}) w$ in $D$, and $w(x, 0)=f(x)$ on $\sigma$.

Proof of Theorem 4.2. According to Theorem 2.1, we again have integral representation (4.3) for $w_{i}(z)$, but here $\phi_{1}(z), \phi_{2}(\zeta)$ are holomorphic for $z \in D$, $\zeta \in \bar{D}$, and continuous for $z \in D \cup \sigma, \zeta \in \bar{D} \cup \sigma$.

Therefore, for $x \in \sigma$, we again have (4.4) and (4.5). And they suggest the following integral equations: 


$$
\begin{aligned}
G_{1}(z) \phi_{1}(z) & +\int_{0}^{z} \Gamma_{1,1}\left(z, z, t, \bar{z}_{1}\right) G_{1}(z) \phi_{1}(t) d t \\
& -\int_{0}^{z} \Gamma_{2,2}\left(z, z, z_{2}, t\right) G_{2}(z) \phi_{2}^{*}(t) d t=a(z), \\
G_{2}^{*}(z) \phi_{2}^{*}(z) & +\int_{0}^{z} \Gamma_{1,2}^{*}\left(z, z, t, z_{2}\right) G_{2}^{*}(z) \phi_{2}^{*}(t) d t \\
& -\int_{0}^{z} \Gamma_{2,1}^{*}\left(z, z, \bar{z}_{1}, t\right) G_{1}^{*}(z) \phi_{1}(t) d t=b(z),
\end{aligned}
$$

for the unknown functions $\phi_{1}(z), \phi_{2}^{*}(z)$ in $\bar{D} \cup \sigma$, where

$$
\begin{aligned}
a(z)= & G_{2}(z) \phi_{2}(z)+\int_{z_{2}}^{z} \Gamma_{1,2}\left(z, z, t, \bar{z}_{2}\right) G_{2}(z) \phi_{2}(t) d t \\
& +\int_{\bar{z}_{2}}^{0} \Gamma_{2,2}\left(z, z, z_{2}, t\right) G_{2}(z) \phi_{2}^{*}(t) d t \\
& -\int_{z_{1}}^{0} \Gamma_{1,1}\left(z, z, t, \bar{z}_{1}\right) G_{1}(z) \phi_{1}(t) d t \\
& +G_{2}(z) U_{0,2}(z, z)-G_{1}(z) U_{0,1}(z, z) \\
& +\int_{\bar{z}_{1}}^{z} \Gamma_{2,1}\left(z, z, z_{1}, t\right) G_{1}(z) \phi_{1}^{*}(t) d t, \\
b(z)= & G_{1}^{*}(z) \phi_{1}^{*}(z)+\int_{z_{1}}^{z} \Gamma_{1,1}^{*}\left(z, z, t, z_{1}\right) G_{1}^{*}(z) \phi_{1}^{*}(t) d t \\
& -\int_{\bar{z}_{2}}^{0} \Gamma_{1,2}^{*}\left(z, z, t, z_{2}\right) G_{2}^{*}(z) \phi_{2}^{*}(t) d t \\
& +\int_{z_{1}}^{0} \Gamma_{2,1}^{*}\left(z, z, \bar{z}_{1}, t\right) G_{1}^{*}(z) \phi_{1}(t) d t \\
& +G_{1}^{*}(z) U_{0,1}^{*}(z, z)-G_{2}^{*}(z) U_{0,2}^{*}(z, z) \\
& -\int_{z_{2}}^{z} \Gamma_{2,2}^{*}\left(z, z, \bar{z}_{2}, t\right) G_{2}^{*}(z) \phi_{2}(t) d t .
\end{aligned}
$$

Therefore, the integral equations (4.8) and (4.9) have the unique solution $\left(\phi_{1}(z), \phi_{2}^{*}(z)\right)$ which is holomorphic in $\bar{D}$, continuous in $\bar{D} \cup \sigma$, and coincides with the given $\left(\phi_{1}(z), \phi_{2}^{*}(z)\right)$ on $\sigma$.

It therefore follows that $\phi_{1}(z), \phi_{2}(z)$ can be considered as holomorphic functions in $D \cup \sigma \cup \bar{D}$. By Theorem 1.1, $w_{1}(z)$ and $w_{2}(z)$ are solutions of (4.1) and (4.2) in $D \cup \sigma \cup \bar{D}$ respectively. This completes the proof.

Corollary 4.1. Let $w(x, y)$ be a solution of (1.1) in $D, C^{\prime}$ in $D \cup \sigma, f(z)$ be holomorphic in $D, C^{\prime}$ in $D \cup \sigma$, such that $w(x, 0)=f(x)$ on $\sigma$. If $B(z, z) \neq 0$ for $z \in \bar{D}$, then $f(z)$ is holomorphic in $D \cup \sigma \cup \bar{D}$, and $w(x, y)$ can be continued analytic into whole $D \cup \sigma \cup \bar{D}$ as a solution of (1.1).

The following corollary of Theorem 4.2 is a generalization of a well-known criterion for the analytic continuation of a holomorphic function. 
Corollary 4.2. If $\rho(x)$ is a Cauchy data of a solution $w_{1}(x, y)$ of $(1.1)$ in $D$, and is a Cauchy data of a solution $w_{2}(x, y)$ of $(1.1)$ in $\bar{D}$, then there is a unique solution $w(x, y)$ of $(1.1)$ in $D \cup \sigma \cup \bar{D}$ such that

$$
\begin{aligned}
w(x, y) & =w_{1}(x, y) & & \text { in } D \\
& =w_{2}(x, y) & & \text { in } \bar{D} .
\end{aligned}
$$

Proof of Corollary 4.1 and Corollary 4.2. These are immediate consequences of Theorem 4.1 and Theorem 4.2.

Let $f(z)$ be a function defined on a rectifiable simple Jordan curve $\Gamma$. This function may be regarded as a function of the length of the arc $s$, i.e., $f(z(s))=f(s)$. If $f(s)$ is continuous with its derivatives up to the $m$ th order on the arc $0 \leq s \leq l, f$ will be said to belong to the class $C^{m}(\Gamma)$. If, moreover, $f^{(m)}(s)$ satisfies the Hölder condition with an index $\mu, 0<\mu<1$, then it will be said that $f \in C_{\mu}^{m}(\Gamma)$.

We now state a lemma which is proved by Vekua [7].

Lemma 4.1 (Vekua [7, p. 313]). Let $G$ be a simply connected domain such that $G \cup \partial G \subset D \cup \sigma \cup \bar{D}$, whose boundary $\partial G$ is a simple smooth curve. Let $w(x, y)$ be a solution of differential equation (1.1) in $G$, continuous in $G \cup \partial G$, satisfying $\alpha u+\beta v=\operatorname{Re}[\overline{\lambda(z)} w]=\rho(z), \lambda=\alpha+i \beta$ on $\partial G$, where $\rho$ and $\lambda \in C_{\mu}^{\prime}(\partial G)$, $0<\mu<1,|\lambda(z)|=1$ on $\partial G$. Then $\partial w / \partial x, \partial w / \partial y$ are Hölder continuous with index $\mu$ on $G \cup \partial G$.

The next corollary is a refinement of Theorem 4.1.

Corollary 4.3. Let $\rho(x)$ be a Cauchy data for a solution $w_{1}(x, y)$ of $(4.1)$ in $D$ and for a solution $w_{2}(z, y)$ of $(4.2)$ in $D$, and $\rho^{\prime}(x)$ be Hölder continuous with index $\mu$, $0<\mu<1$, on $\sigma$. If $B_{1}(x, x) \not \equiv B_{2}(x, x)$ on $\sigma$, then $\rho(x)$ is analytic on $\sigma$, and its analytic continuation $\rho(z)$ is holomorphic in $D \cup \sigma \cup \bar{D}$, except the zeros of $B_{1}^{*}(z, z)-B_{2}^{*}(z, z)$ in $\bar{D}$. Furthermore, $w_{1}(x, y)$ and $w_{2}(x, y)$ can be continued analytically into whole $D \cup \sigma \cup \bar{D}$, except the zeros of $B_{1}^{*}(z, z)-B_{2}^{*}(z, z)$ in $D$, as solutions of (4.1) and (4.2) respectively.

Proof. Since $\rho^{\prime}(x)$ is Hölder continuous with index $\mu, 0<\mu<1$, on $\sigma$, it follows from Lemma 4.1 that $\partial w_{1} / \partial x, \partial w_{2} / \partial x, \partial w_{1} / \partial y$, and $\partial w_{2} / \partial y$ are Hölder continuous in $D \cup \sigma$. Thus the desired result follows immediately from Theorem 4.1. This completes the proof.

If we further assume that $\rho(x)$ is nowhere analytic on $\sigma$, then Theorem 4.1, Theorem 4.2 and Corollary 4.3 imply the following:

Theorem 4.3. Let $\rho(x)$ be nowhere analytic on $\sigma$ such that $\rho^{\prime}(x)$ is Hölder continuous with index $\mu, 0<\mu<1$, on $\sigma$, and $B_{1}(x, x) \not \equiv B_{2}(x, x)$ on $\sigma$. If $\rho(x)$ is a Cauchy data of a solution $w(x, y)$ of $(4.2)$ in $D$, then the Cauchy problem with data $\rho(x)$ for equation (4.1) has no solution in $D$. 
Theorem 4.4. Let $\rho(x)$ be nowhere analytic on $\sigma$. If $\rho(x)$ is a Cauchy data of a solution $w(x, y)$ of $(4.2)$ in $\bar{D}$, then the Cauchy problem with data $\rho(x)$ for equation (4.1) has no solution in $D$.

\section{REFERENCES}

1. L. Bers, Theory of pseudo-analytic functions, Institute for Math. and Mechanics, New York Univ., New York, 1953. MR 15, 211.

2. L. Graves, The theory of functions of real variables, McGraw-Hill, New York, 1965.

3. P. Henrici, $A$ survey of $I$. N. Vekua's theory of elliptic partial differential equations with analytic coefficients, Z. Angew. Math. Phys. 8 (1957), 169-203. MR 19, 38.

4. P. R. Garabedian, Analyticity and reflection for plane elliptic systems, Comm. Pure Appl. Math. 14 (1961), 315-322. MR 25 \#309.

5. H. Lewy, On the reflection laws of second order differential equations in two independent variables, Bull. Amer. Math. Soc. 65 (1959), 37-58. MR 21 \#2810.

6. - A note on harmonic functions and a hydrodynamical application, Proc. Amer. Math. Soc. 3 (1952), 111-113. MR 14, 168.

7. I. N. Vekua, Generalized analytic functions, Fizmatgiz, Moscow, 1959; English transl., Pergamon Press, London and Addison-Wesley, Reading, Mass., 1962. MR 21 \# 7288; MR 27 \#321.

8. - New methods for solving elliptic equations, OGIZ, Moscow, 1948; English transl., Series in Appl. Math., vol. 1, North-Holland, Amsterdam; Interscience, New York, 1967. MR 11, 598; MR 35 \#3243.

9. C. L. Yu, Reflection principle for systems of first order elliptic equations with analytic coefficients, Trans. Amer. Math. Soc. 164 (1972), 489-501.

Department of Mathematics, Florida State University, Tallahassee, Florida 32306 